\title{
Performance Evaluation of 4D Reconstruction Methods for Gated Cardiac Single Photon Emission Computed Tomography in Obese Patients
}

\author{
S Sayeram $^{1}$, DS Lalush ${ }^{2}$ \\ ${ }^{1}$ University of North Carolina at Chapel Hill, Chapel Hill, NC, USA \\ ${ }^{2}$ North Carolina State University, Raleigh, NC, USA
}

\begin{abstract}
The purpose of this study is to evaluate $4 D$ reconstruction methods for the processing of gated cardiac single photon emission computed tomography (SPECT) images from obese patients.

Clinical gated SPECT projection data were reconstructed using the ordered-subsets expectationmaximization (OS-EM) and the $4 D$ rescaled blockiterative maximum a posteriori (RBI-MAP) reconstruction algorithms. The data were used to derive a relative distribution of quantitative tetrofosmin uptake ratios for the various organs. Using anthropometric data, body sizes were determined for a standard obese male and female NURBS-based cardiac torso (NCAT) phantom. Tetrofosmin uptake ratios were modeled in the obese phantoms and projection data were generated. Poisson noise was simulated, and the noisy data were reconstructed with OS-EM and $4 D$ RBI-MAP.

The projected and reconstructed data from the obese phantoms make for a realistic model of tetrofosmin uptake distribution. Qualitatively, 4D processing methods substantially improve visualization of cardiac motion in these very noisy images. We conclude that the tetrofosmin patient models provide a realistic, controllable test vehicle on which to evaluate $4 D$ processing methods for gated cardiac SPECT. $4 D$ processing methods improve the quality of gated SPECT images in obese patients, providing a usable representation of cardiac motion in these difficult-to-image patients.
\end{abstract}

\section{Introduction}

Myocardial SPECT imaging is frequently the imaging technique of choice used to visualize perfusion defects of the heart. Cardiac gating adds a vital dimension to myocardial imaging, enabling the diagnostician to visualize cardiac motion and wall thickening, and assess cardiac function quantitatively by means of chamber volumes calculated by clinical software packages. A principal problem with gated SPECT images is the increase in noise due to the division of acquired counts into time frames, and this issue is magnified in the case of obese patients, due to additional attenuation from the larger body size. The images can be noisy enough for clinicians to discount 4D data altogether in this population of patients.

Our hypothesis is that $4 \mathrm{D}$ reconstruction methods applied to gated studies in this population will result in more usable images with less noise degradation and no loss of temporal resolution as compared to images smoothed with 2D and 3D Fourier filters. The rescaled block-iterative maximum a posteriori (RBI-MAP) algorithm is a modified version of the iterative RBI-EM algorithm, closely related to the ordered-subset, expectation-maximization (OS-EM) algorithm [1]. The former incorporates useful features of the MAP reconstruction method (particularly by the use of Gibbs priors [2], which are designed to retain edge features while smoothing noise, thus contributing to better temporal resolution when applied in the time dimension [3]). It is not known whether 4D reconstruction methods result in improved image quality in reconstructed gated SPECT images from obese patients, as compared to 3D reconstruction methods. Thus, the aim of this study is to evaluate this question by means of computer simulation tools described herein.

\section{Methods}

Anthropometric data was used to obtain a distribution of body dimensions across different populations. This information was used to determine an appropriate body size to use in building a standard obese male and female non-uniform rational B-spline-based (NURBS) cardiac torso (NCAT) phantom. The NCAT phantom is a computerized phantom which includes models of cardiac and respiratory motion, allows for the modeling of perfusion and motion defects of the myocardium, for the modeling of different organ and body sizes, as well as for the modification of radioactivity uptake ratios and attenuation maps [4], [5]. It is thus a very powerful tool that provides the researcher with a gold standard on which to test the efficacy of different reconstruction 
methods or other parameters.

Clinical gated SPECT data were obtained from the Nuclear Medicine clinic at UNC Hospital, and reconstructed with the $3 \mathrm{D}$ OS-EM and 4D RBI-MAP algorithms. The patients had been administered technetium Tc-99m tetrofosmin, and images had been acquired in matrix sizes of $64 \times 64$, with 64 views gated into 8 frames. The projection data and reconstructed data were used to derive relative distributions of quantitative uptake ratios for various organs.

Using this information and published literature on tetrofosmin uptake kinetics, several uptake distributions were modeled in the organs of the obese 4D NCAT phantoms. The noise levels in the patient data were also used to determine realistic noise levels to model in the NCAT phantom population. Tetrofosmin uptake ratios were then modeled in the obese phantoms, and projection data including the effects of non-uniform attenuation, detector response and scatter, were generated from the obese male and female phantoms, in matrix sizes of $64 \times 64$, with 64 views over $180^{\circ}$, in 8 bins representing the phases of the cardiac cycle (as these parameters are typical of clinical acquisitions at our institution). Poisson noise was simulated, and the noisy data were reconstructed with OS-EM and 4D RBI-MAP, and no post-reconstruction filtering was done.

The projection data and reconstructed data from the patients were compared to those from the phantoms, and the images reconstructed with 3D OS-EM were compared to those reconstructed with 4D RBI-MAP, to evaluate subjectively the image quality in terms of image contrast and noise.

From each of the reconstructed sets of images of the patient and the phantom, the central slice of the heart image was extracted, and the same views from each of the 8 time frames were compiled into a movie in audio video interleave (AVI) format. These were then subjectively compared to judge the fidelity of cardiac motion preserved by the different reconstruction methods.

\section{Results}

Selected transaxial slices of attenuation maps and tetrofosmin activity distributions of the standard obese male and female phantoms are shown below in Figures 1 and 2.

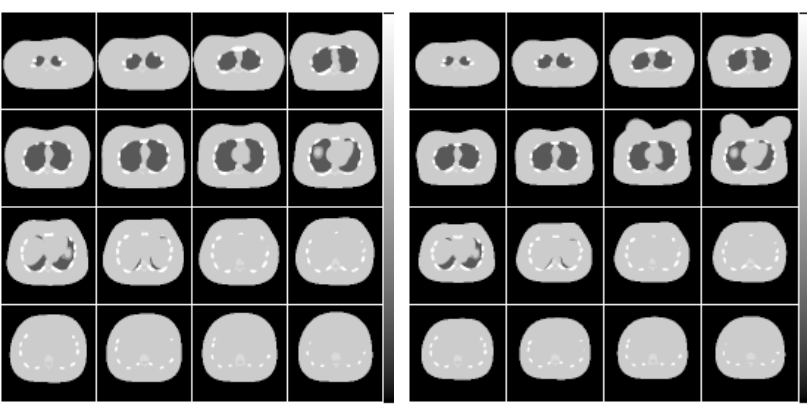

Figure 1: Selected transaxial slices showing the attenuation maps of the obese male and female phantom in matrix sizes of $64 \times 64$, with pixel sizes of $0.5 \mathrm{~cm}$
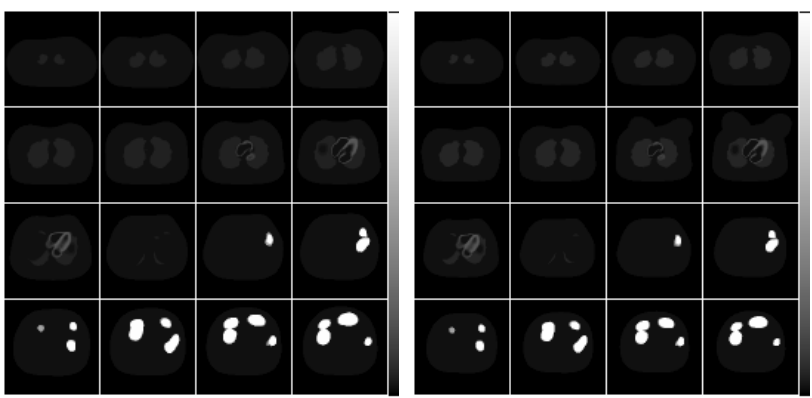

Figure 2: Selected transaxial slices showing the activity maps of the obese male and female phantom, modeling a Tc-99m tetrofosmin activity distribution in matrix sizes of $64 \times 64$, with pixel sizes of $0.5 \mathrm{~cm}$; the average of 8 bins is shown

Figures 3 and 4 show selected views of projection data from the obese patient and the standard obese male phantom, and they are very similar in appearance to one another in terms of organ radiopharmaceutical uptake. There is heightened uptake in the intestines combined with rapid clearance from the liver as is typical for tetrofosmin studies.

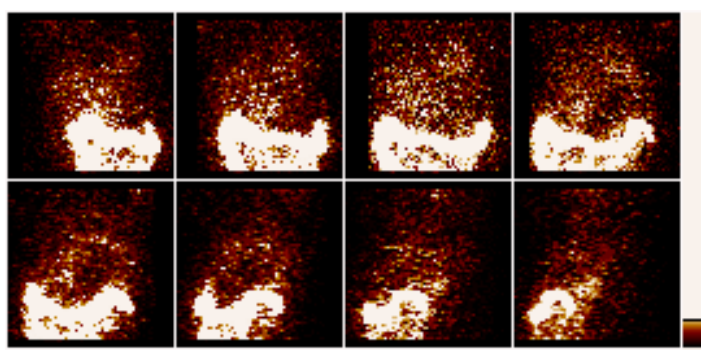

Figure 3: Selected views of projection data from the stress image of an obese patient who was administered Tc-99mTetrofosmin; this dataset shows the first frame of the cardiac cycle 


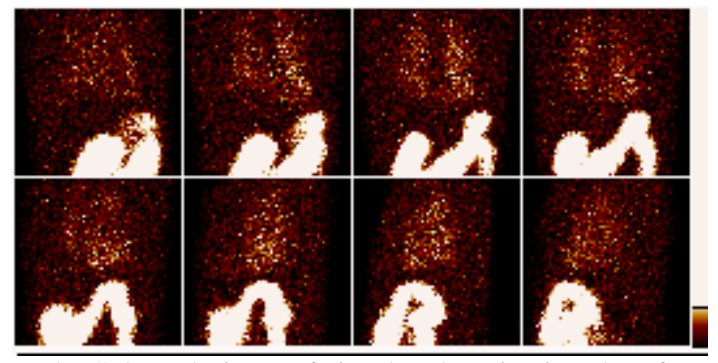

Figure 4: Selected views of simulated projection data from the obese male phantom simulating Tc-99m tetrofosmin uptake

Reconstructed images from the obese patient are seen in Figure 5, while reconstructed images from the obese male phantom are depicted in Figure 6. In both sets of images, the images reconstructed with the 4D RBI-MAP algorithm show better image contrast and less noise degradation, than images reconstructed with the 3D OSEM method.
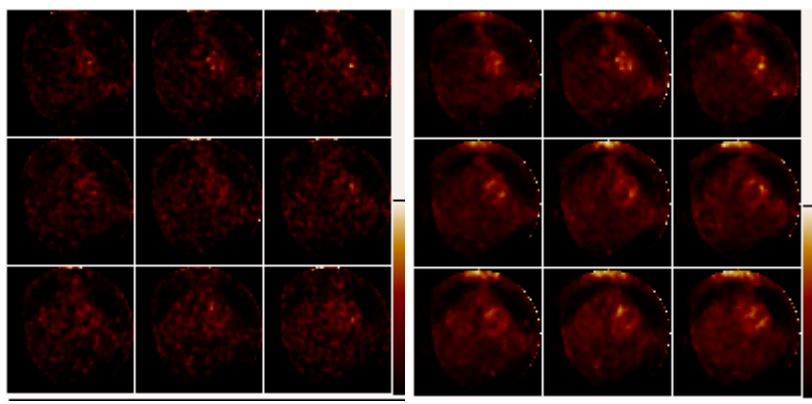

Figure 5: Selected transaxial slices of reconstructed images from an obese patient who was administered Tc-99mTetrofosmin; the iterative 3D OS-EM and the 4D RBI-MAP reconstruction algorithms were used, and the $3^{\text {rd }}$ iteration of the reconstructed images showing the $1^{\text {st }}$ of 8 gates is seen
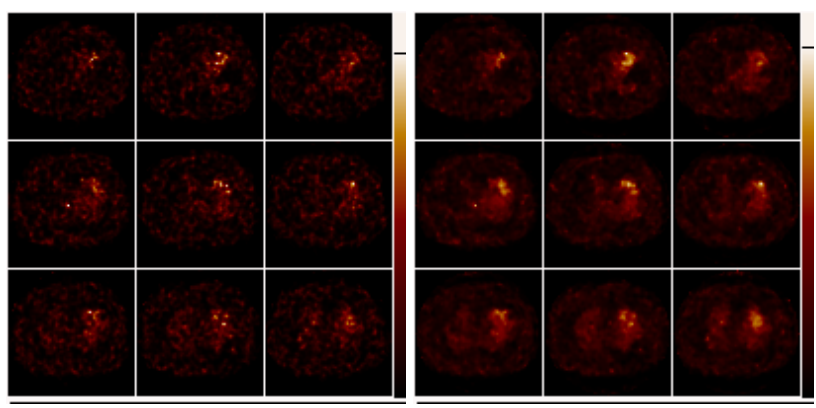

Figure 6: Selected transaxial slices of reconstructed images from the obese male phantom; the iterative 3D OS-EM and the 4D RBI-MAP reconstruction algorithms were used, and the $3^{\text {rd }}$ iteration of the reconstructed images showing the $1^{\text {st }}$ of 8 gates is seen

Observation of the AVI loops reveals that RBI-MAP provides a more contiguous representation of the myocardium, as compared to the noisy, broken appearance of the non-MAP results. This results in a better presentation of motion, allowing the observer to visualize motion more completely. A motion observer study [6] is required to conclusively evaluate these properties.

\section{Discussion and conclusions}

The standard obese male and female phantoms generated in this study appear to be a realistic model of tetrofosmin activity distribution, and can be used as a test vehicle to evaluate the performance of various reconstruction methods for $4 \mathrm{D}$ gated myocardial SPECT studies.

The 4D RBI-MAP reconstruction algorithm results in images with better fidelity of motion than the 3D OS-EM algorithm; this is key in gated studies as they are primarily performed to estimate cardiac motion and volumes as precisely as possible.

Further studies will be undertaken to evaluate whether there is a measurable difference in the quantitative accuracy (in estimating cardiac volumes) between the two reconstruction methods, and to gauge which is a better estimator of cardiac wall motion. A motion observer study will be useful towards this end [6].

\section{References}

[1] Hudson HM, and Larkin RS. Accelerated image reconstruction using ordered subsets of projection data. IEEE Trans Med Imag. 1994; 13(4): 601-609.

[2] Lalush DS, and Tsui BMW. A generalized Gibbs prior for maximum a posteriori reconstruction in SPECT. Phys Med Biol. 1993; 38: 729-741.

[3] Lalush DS, and Tsui BMW. Block-iterative techniques for fast $4 \mathrm{D}$ reconstruction using a priori motion models in gated cardiac SPECT. Phys Med Biol. 1998; 43: 875-887.

[4] Segars WP, Lalush DS, and Tsui BMW. Modeling respiratory mechanics in the MCAT and spline-based MCAT phantoms. IEEE Trans Nucl Sci. 2001; 48(1): 8997.

[5] Segars WP, and Tsui BMW. Study of the efficacy of respiratory gating in the myocardial SPECT using the new 4D NCAT phantom. IEEE Trans Nucl Sci. 2002; 49(3): 675-679.

[6] Lalush DS, Jatko M, and Segars WP. An observer study methodology for evaluating detection of motion abnormalities in gated myocardial perfusion SPECT. IEEE Trans Biomed Eng. 2005; 52(3): 480-485.

Address for correspondence

Sunita Sayeram

Joint Dept. of Biomedical Engineering, UNC-CH

152 MacNider Hall, CB \#7575

Chapel Hill, NC 27599-7575

E-mail: ssayeram@bme.unc.edu 
